\title{
DYNAMICS OF SPATIAL LOGISTIC MODEL: FINITE SYSTEMS
}

\author{
YURI KOZITSKY
}

\begin{abstract}
The spatial logistic model is a system of point entities (particles) in $\mathbb{R}^{d}$ which reproduce themselves at distant points (dispersal) and die, also due to competition. The states of such systems are probability measures on the space of all locally finite particle configurations. In this paper, we obtain the evolution of states of 'finite systems', that is, in the case where the initial state is supported on the subset of the configuration space consisting of finite configurations. The evolution is obtained as the global solution of the corresponding Fokker-Planck equation in the space of measures supported on the set of finite configurations. We also prove that this evolution preserves the existence of exponential moments and the absolute continuity with respect to the Lebesgue-Poisson measure.
\end{abstract}

\section{INTRODUCTION}

In a number of applications, one deals with large systems of interacting entities evolving in time and distributed over an unbounded continuous habitat. In simple models of such systems, each entity is completely characterized by its spatial location $x \in \mathbb{R}^{d}, d \geq 1$. The entities form discrete sets, typically described by probability distributions. Therefore, the proper mathematical context for studying models of this kind is the theory of random point fields on $\mathbb{R}^{d}$, cf. [13, page 1311]. In this approach, the phase space is the set of point configurations

$$
\Gamma=\left\{\gamma \subset \mathbb{R}^{d}:|\gamma \cap \Lambda|<\infty \text { for any compact } \Lambda \subset \mathbb{R}^{d}\right\},
$$

where $|\cdot|$ denotes cardinality. A proper subset of $\Gamma$ is the set of finite configurations $\Gamma_{0}=\left\{\gamma \in \Gamma:|\gamma| \in \mathbb{N}_{0}\right\}$. The set $\Gamma$ can be equipped with the vague topology, see [1, 10], and thus with the corresponding Borel $\sigma$-field $\mathcal{B}(\Gamma)$. The elements of $\Gamma$ are considered as point states in the sense that, for a suitable function $F: \Gamma \rightarrow \mathbb{R}$, the number $F(\gamma)$ is the value of observable $F$

1991 Mathematics Subject Classification. 82C22, 92D25, 60J80.

Key words and phrases. Individual-based model; birth-and-death process; random point field.

Supported by the DFG through SFB 701: "Spektrale Strukturen und Topologische Methoden in der Mathematik", by the ZiF Research Group "Stochastic Dynamics: Mathematical Theory and Applications" (Universität Bielefeld), and by the European Commission under the project STREVCOMS PIRSES-2013-612669. 
in state $\gamma$. Along with such point states one employs those determined by probability measures on $\mathcal{B}(\Gamma)$. Then the corresponding value is the integral

$$
\langle\langle F, \mu\rangle\rangle:=\int_{\Gamma} F d \mu,
$$

and the system's dynamics are described as maps $\mu_{0} \mapsto \mu_{t}, t>0$. The elementary acts here include birth, death, immigration, jumps, diffusion, etc. In the Markov approach, the map $\mu_{0} \mapsto \mu_{t}$ is obtained from the FokkerPlanck equation

$$
\frac{d}{d t} \mu_{t}=L^{\mu} \mu_{t},\left.\quad \mu_{t}\right|_{t=0}=\mu_{0}, \quad t \geq 0,
$$

in which 'operator' $L^{\mu}$ specifies the model. By the duality

$$
\left\langle\left\langle F_{0}, \mu_{t}\right\rangle\right\rangle=\left\langle\left\langle F_{t}, \mu_{0}\right\rangle\right\rangle, \quad t>0,
$$

the observed evolution $\left\langle\left\langle F, \mu_{0}\right\rangle\right\rangle \mapsto\left\langle\left\langle F, \mu_{t}\right\rangle\right\rangle$ can also be considered as the evolution $\left\langle\left\langle F_{0}, \mu\right\rangle\right\rangle \mapsto\left\langle\left\langle F_{t}, \mu\right\rangle\right\rangle$, obtained from the Kolmogorov equation

$$
\frac{d}{d t} F_{t}=L F_{t},\left.\quad F_{t}\right|_{t=0}=F_{0},
$$

where $L$ and $L^{\mu}$ are dual in the sense of (1.4). A number of such models are discussed in [8]. In this article, we deal with the spatial logistic model specified by

$$
\begin{array}{r}
(L F)(\gamma)=\sum_{x \in \gamma}\left[m+E^{-}(x, \gamma \backslash x)\right][F(\gamma \backslash x)-F(\gamma)] \\
+\int_{\mathbb{R}^{d}} E^{+}(y, \gamma)[F(\gamma \cup y)-F(\gamma)] d y,
\end{array}
$$

where

$$
E^{+}(x, \gamma)=\sum_{y \in \gamma} a^{+}(x-y) .
$$

The first term in (1.5) describes the death of the particle located at $x$ occurring independently with rate $m \geq 0$ (intrinsic mortality), and under the influence of the other particles in $\gamma$ with rate $E^{-}(x, \gamma \backslash x) \geq 0$ (competition). The second term in (1.5) describes the birth of a particle at $y \in \mathbb{R}^{d}$ given by the whole configuration $\gamma$ with rate $E^{+}(y, \gamma) \geq 0$. Models of this kind appear in population biology, see [9, 14 for more detail. A particular case of (1.5) with $E^{-} \equiv 0$ is the contact model [7, 11, 12].

The sums in (1.5) and (1.6) are taken over possibly infinite sets, and thus the very definition of $L^{\mu}$ in (1.3) gets highly problematic if one wants to include in its domain nontrivial states with 'infinite number of entities'. For the same reason, direct solving (1.3) for such states is also beyond the possibilities of the existing mathematical methods. For infinite systems of physical particles, N. N. Bogoliubov suggested studying the dynamics of states indirectly - by means of correlation functions, see [2. Their evolution is obtained from an infinite chain of equations, so called $B B G K Y$ hierarchy, 
that links to each other correlation functions of different order. Starting from the late 1990'th, a similar approach is being implemented in studying Markov dynamics on the phase space $\Gamma$, see [6, 14] and the references quoted therein. In this approach, various aspects of the evolution of the model specified by a particular case of (1.5) with 'infinite number of entities' were studied in [4, 5, 6]. The results of [4] were announced in [3]. The aim of this work is to describe the evolution of states of the model (1.5) with 'finite number of entities'.

\section{The Setup And Results}

2.1. Preliminaries. By $\mathcal{B}\left(\mathbb{R}^{d}\right)$ and $\mathcal{B}_{\mathrm{b}}\left(\mathbb{R}^{d}\right)$ we denote the set of all Borel and all bounded Borel subsets of $\mathbb{R}^{d}$, respectively. The set of configurations $\Gamma$ defined in (1.1) is equipped with the vague topology - the weakest topology that makes the maps

$$
\Gamma \ni \gamma \mapsto \sum_{x \in \gamma} f(x) \in \mathbb{R}
$$

continuous for all compactly supported continuous functions $f: \mathbb{R} \rightarrow \mathbb{R}$. This topology can be completely and separably metrized that turns $\Gamma$ into a Polish spaces, see [1, 10]. By $\mathcal{B}(\Gamma)$ and $\mathcal{P}(\Gamma)$ we denote the Borel $\sigma$-field of subsets of $\Gamma$ and the set of all probability measures on $\mathcal{B}(\Gamma)$, respectively.

The set of finite configurations

$$
\Gamma_{0}=\bigcup_{n \in \mathbb{N}_{0}} \Gamma^{(n)}
$$

is the disjoint union of the sets of $n$-particle configurations:

$$
\Gamma^{(0)}=\{\emptyset\}, \quad \Gamma^{(n)}=\{\gamma \in \Gamma:|\gamma|=n\}, \quad n \in \mathbb{N} .
$$

For $n \geq 2, \Gamma^{(n)}$ can be identified with the symmetrization of the set

$$
\left\{\left(x_{1}, \ldots, x_{n}\right) \in\left(\mathbb{R}^{d}\right)^{n}: x_{i} \neq x_{j} \text {, for } i \neq j\right\},
$$

which allows one to introduce the corresponding (Euclidean) topology on $\Gamma^{(n)}$. Then the topology on the whole $\Gamma_{0}$ is defined as follows: $A \subset \Gamma_{0}$ is said to be open if its intersection with each $\Gamma^{(n)}$ is open. This (intrinsic) topology differs from that induced on $\Gamma_{0}$ by the vague topology of $\Gamma$. At the same time, $\Gamma_{0} \in \mathcal{B}(\Gamma)$. Thus, a function $G: \Gamma_{0} \rightarrow \mathbb{R}$ is measurable as a function on $\Gamma$ if and only if its restrictions to each $\Gamma^{(n)}$ are Borel functions. Clearly, these restrictions fully determine $G$. In view of (2.1), the restriction of $G$ to $\Gamma^{(n)}$ can be extended to a symmetric Borel function $G^{(n)}:\left(\mathbb{R}^{d}\right)^{n} \rightarrow \mathbb{R}$, $n \in \mathbb{N}$, such that

$$
G(\gamma)=G^{(n)}\left(x_{1}, \ldots, x_{n}\right), \quad \text { for } \gamma=\left\{x_{1}, \ldots, x_{n}\right\} .
$$

It is convenient to complement (2.2) by putting $G(\emptyset)=G^{(0)} \in \mathbb{R}$. Let all $G^{(n)}, n \in \mathbb{N}$, be continuous functions with compact support and $G$ be as in 
(2.2). For all such $G$, we then write

$$
\int_{\Gamma_{0}} G(\gamma) \lambda(d \gamma)=G^{(0)}+\sum_{n=1}^{\infty} \frac{1}{n !} \int_{\left(\mathbb{R}^{d}\right)^{n}} G^{(n)}\left(x_{1}, \ldots x_{n}\right) d x_{1} \cdots d x_{n} .
$$

This expression determines a $\sigma$-finite measure $\lambda$ on $\Gamma_{0}$, called the LebesguePoisson measure.

Having in mind that $\Gamma_{0} \in \mathcal{B}(\Gamma)$ we define

$$
\mathcal{F}\left(\Gamma_{0}\right)=\left\{B \subset \Gamma_{0}: B \in \mathcal{B}(\Gamma)\right\} .
$$

The intrinsic topology of $\Gamma_{0}$ is clearly metrizable. The corresponding metric space is also complete and separable. The embedding $\Gamma_{0} \hookrightarrow \Gamma$ is continuous and hence $\mathcal{B}\left(\Gamma_{0}\right) / \mathcal{B}(\Gamma)$-measurable. By the Kuratowski theorem 15, page 21] we then have

$$
\mathcal{F}\left(\Gamma_{0}\right)=\mathcal{B}\left(\Gamma_{0}\right) .
$$

Thus, each $\mu \in \mathcal{P}(\Gamma)$ with the property

$$
\mu\left(\Gamma_{0}\right)=1
$$

can be redefined as a probability measure on $\mathcal{B}\left(\Gamma_{0}\right)$.

The considered system of entities is finite if it is in a state with property (2.4). In the probabilistic interpretation, such a system has a 'random number of entities', whereas $\mu\left(\Gamma^{(n)}\right)$ is the probability that this number is $n$. Correspondingly,

$$
N_{\mu}:=\int_{\Gamma_{0}}|\gamma| \mu(d \gamma)=\sum_{n=0}^{\infty} n \mu\left(\Gamma^{(n)}\right)
$$

is the expected number of entities in state $\mu$. Of course, the convergence in (2.5) depends on the asymptotic properties of the sequence $\left\{\mu\left(\Gamma^{(n)}\right)\right\}_{n \in \mathbb{N}_{0}}$, which characterize the state. For the Poisson measure $\pi_{\varrho}$ with density $\varrho$, we have

$$
N_{\pi_{\varrho}}=\int_{\mathbb{R}^{d}} \varrho(x) d x
$$

and

which yields

$$
\pi_{\varrho}\left(\Gamma^{(n)}\right)=N_{\pi_{\varrho}}^{n} \exp \left(-N_{\pi_{\varrho}}\right) / n !
$$

$$
\int_{\Gamma_{0}} \exp (\beta|\gamma|) \pi_{\varrho}(d \gamma)=\exp \left(\left(e^{\beta}-1\right) \int_{\mathbb{R}^{d}} \varrho(x) d x\right),
$$

holding for all $\beta \geq 0$. Another relevant property of $\pi_{\varrho}$ is that it is absolutely continuous with respect to the Lebesgue-Poisson measure introduced in (2.3). Furthermore,

$$
\pi_{\varrho}(d \gamma)=\left(\prod_{x \in \gamma} \varrho(x)\right) \exp \left(-N_{\pi_{\varrho}}\right) \lambda(d \gamma) .
$$

The aim of this work is to show that the evolution described by (1.3) and (1.5) can preserve the properties of having finite exponential moments as 
in (2.6) and of being absolutely continuous with respect to the LebesguePoisson measure. We do this in Theorem 2.2 below.

2.2. The results. For finite systems, one can restrict the sums in (1.5) and (1.6) to finite $\gamma$, which in turn allows for writing the Fokker-Planck equation (1.3) in the form

$$
\begin{aligned}
\frac{d}{d t} \mu_{t}(d \eta) & =\left(L^{\mu} \mu\right)(d \eta) \\
& =-\Xi\left(\mathbb{R}^{d}, \eta\right) \mu_{t}(d \eta)+\int_{\Gamma_{0}} \Xi(d \eta, \gamma) \mu_{t}(d \gamma),\left.\quad \mu_{t}\right|_{t=0}=\mu_{0}
\end{aligned}
$$

where, for $A \in \mathcal{B}\left(\Gamma_{0}\right)$ and $\gamma \in \Gamma_{0}$,

$$
\left.\Xi(A, \gamma)=\sum_{x \in \gamma}\left(m+E^{-}(x, \gamma \backslash x)\right) \mathbb{I}_{A}(\gamma \backslash x)+\int_{\mathbb{R}^{d}} E^{+}(y, \gamma)\right) \mathbb{I}_{A}(\gamma \cup y) d y,
$$

which is a measure kernel on $\left(\Gamma_{0}, \mathcal{B}\left(\Gamma_{0}\right)\right)$. The kernels in (1.6), and hence in (2.9), are supposed to satisfy: (i) $E^{-}$is nonnegative and measurable in each of its arguments; (ii) $a^{+}(x)=a^{+}(-x) \geq 0$ and

$$
a^{+} \in L^{1}\left(\mathbb{R}^{d}\right) \text {. }
$$

Set

$$
\left\langle a^{+}\right\rangle=\int_{\mathbb{R}^{d}} a^{+}(x) d x, \quad E^{-}(\gamma)=\sum_{x \in \gamma} E^{-}(x, \gamma \backslash x)
$$

Then

$$
0 \leq \Xi\left(\mathbb{R}^{d}, \gamma\right)=m|\gamma|+E^{-}(\gamma)+\left\langle a^{+}\right\rangle|\gamma|<\infty, \quad \gamma \in \Gamma_{0} .
$$

Now let $\mathcal{M}$ be the set of all finite signed measures (i.e., $\sigma$-additive functions) on $\mathcal{B}\left(\Gamma_{0}\right)$, and let $\mathcal{M}_{+}$be its subset consisting of nonnegative measures. By means of the Jordan decomposition $\mu=\mu_{+}-\mu_{-}, \mu_{ \pm} \in \mathcal{M}_{+}$, we introduce the norm

$$
\|\mu\|=\mu_{+}\left(\Gamma_{0}\right)+\mu_{-}\left(\Gamma_{0}\right),
$$

which makes $\mathcal{M}$ a Banach space. The right-hand side of (2.8) defines an unbounded linear operator $L^{\mu}: \mathcal{M} \rightarrow \mathcal{M}$ with domain

$$
\mathcal{D}\left(L^{\mu}\right)=\left\{\mu \in \mathcal{M}: \Xi\left(\mathbb{R}^{d}, \cdot\right) \mu_{ \pm} \in \mathcal{M}\right\} .
$$

Definition 2.1. By a classical solution of the problem in (2.8), on the time interval $[0, \tau), \tau \leq+\infty$ and in the space $\mathcal{M}$, we understand a map $[0, \tau) \ni t \mapsto \mu_{t} \in \mathcal{D}\left(L^{\mu}\right)$ which is continuously differentiable on $[0, \tau)$ and such that (2.8) is satisfied for all $t \geq 0$. Such a solution is said to be global if $\tau=+\infty$.

Let us turn now to solving (2.8) in the class of measures absolutely continuous with respect to the Lebesgue-Poisson measure $\lambda$. To this end we set

$$
\mu_{t}(d \gamma)=R_{t}(\gamma) \lambda(d \gamma)
$$


By means of the following standard relation

$$
\int_{\Gamma_{0}}\left(\sum_{x \in \gamma} f(x, \gamma \backslash x)\right) \lambda(d \gamma)=\int_{\Gamma_{0}} \int_{\mathbb{R}^{d}} f(x, \gamma) \lambda(d \gamma) d x
$$

see e.g. [6, Lemma 2.1], one transforms (2.8) into the problem

$$
\begin{aligned}
\frac{d}{d t} R_{t}(\gamma)= & -\left[\sum_{x \in \gamma}\left(m+\left\langle a^{+}\right\rangle+E^{-}(x, \gamma \backslash x)\right)\right] R_{t}(\gamma) \\
+ & \int_{\mathbb{R}^{d}}\left(m+E^{-}(x, \gamma)\right) R_{t}(\gamma \cup x) d x+\sum_{x \in \gamma} E^{+}(x, \gamma \backslash x) R_{t}(\gamma \backslash x), \\
& \left.R_{t}\right|_{t=0}=R_{0} .
\end{aligned}
$$

Correspondingly, the solution of $(2.16)$ is to be sought in the space $L^{1}\left(\Gamma_{0}, \lambda\right)$.

Let $\mathcal{M}_{+, 1}$ denote the set of $\mu \in \mathcal{M}$ such that $\mu \geq 0$ and $\|\mu\|=1$. That is, $\mathcal{M}_{+, 1}$ is the set of all probability measures on $\mathcal{B}\left(\Gamma_{0}\right)$. We shall also use the following sets

$$
\begin{aligned}
\mathcal{M}^{(n)} & =\left\{\mu \in \mathcal{M}: \int_{\Gamma_{0}}|\eta|^{n} \mu_{ \pm}(d \eta)<\infty\right\}, & n \in \mathbb{N}, \\
\mathcal{M}^{\beta} & =\left\{\mu \in \mathcal{M}: \int_{\Gamma_{0}} \exp (\beta|\eta|) \mu_{ \pm}(d \eta)<\infty\right\}, & \beta>0 .
\end{aligned}
$$

Finally, we denote

$$
\mathcal{M}_{+, 1}^{(n)}=\mathcal{M}^{(n)} \cap \mathcal{M}_{+, 1}, \quad \mathcal{M}_{+, 1}^{\beta}=\mathcal{M}^{\beta} \cap \mathcal{M}_{+, 1},
$$

and also

$$
T(\alpha, \beta)=(\alpha-\beta) e^{-\alpha} /\left\langle a^{+}\right\rangle, \quad 0<\beta<\alpha .
$$

Theorem 2.2. The problem in (2.8) with $\mu_{0} \in \mathcal{M}^{(1)}$ has a unique global classical solution $\mu_{t} \in \mathcal{M}$ which has the following properties:

(a) for each $n \in \mathbb{N}, \mu_{0} \in \mathcal{M}_{+, 1}^{(n)}$ implies $\mu_{t} \in \mathcal{M}_{+, 1}^{(n)}$ for all $t>0$;

(b) if the parameters in (2.9) satisfy: (1) $\left\langle a^{+}\right\rangle=0$, or (2) $\left.m>\left\langle a^{+}\right\rangle\right\rangle$ 0 , then $\mu_{0} \in \mathcal{M}_{+, 1}^{\beta}$ implies $\mu_{t} \in \mathcal{M}_{+, 1}^{\beta}$ for all $t>0$, which holds for all positive $\beta$ in case (1) and all $\beta \in\left(0, \log m-\log \left\langle a^{+}\right\rangle\right)$in case (2);

(c) for each $\beta^{*}>0$ and $\beta_{*} \in\left(0, \beta^{*}\right), \mu_{0} \in \mathcal{M}_{+, 1}^{\beta^{*}}$ implies $\mu_{t} \in \mathcal{M}_{+, 1}^{\beta_{*}}$ for all $t \in\left(0, T_{*}\right)$, where $T_{*}=T\left(\beta^{*}, \beta_{*}\right)$, see (2.18);

(d) for all $t>0, \mu_{t}$ has the property (2.15) if $\mu_{0}$ does so.

Let us make some comments on the above statement. Under more restrictive assumptions, part (a) was obtained in [9], see Assumption A and Theorem 3.1, respectively. The properties claimed in (b) - (d) resemble those for the Poisson measure, see (2.6) and (2.7). Note, however, that only in case $(c 1)$ we have the existence of the exponential moments for all $\beta>0$. 
Also note that the only property of $E^{-}$in (2.9) which we need is its measurability, and that the above results hold for $E^{-} \equiv 0$. That is, the competition plays no role if the system is finite, unlike for the same model with infinite number of entities studied in [3, 4].

\section{Proof of Theorem 2.2}

The proof is crucially based on the perturbation theory for semigroups of positive operators developed in [16]. In the next subsection, we present its relevant aspects.

3.1. The Thieme-Voigt perturbation theory. Let $\Psi$ be either $\mathcal{M}$ or $L^{1}\left(\Gamma_{0}, \lambda\right)$. In both cases, the norm $\|\cdot\|_{\Psi}$ is additive on the cone of positive elements $\Psi_{+}$. By $\varphi$ we denote the positive linear functional on $\Psi$ such that $\varphi(\psi)=\|\psi\|_{\Psi}$ whenever $\psi \in \Psi_{+}$. A semigroup $S=\{S(t)\}_{t \geq 0}$ of linear operators on $\Psi$ is called substochastic (respectively, stochastic) if: (a) it is strongly continuous in $t$ ( $C_{0}$-property); (b) $S(t): \Psi_{+} \rightarrow \Psi_{+}$for all $t \geq 0$ (positivity); (c) $\|S(t) \psi\|_{\Psi} \leq\|\psi\|_{\Psi}$ (respectively, $\|S(t) \psi\|_{\Psi}=\|\psi\|_{\Psi}$ ) for all $t \geq 0$ and $\psi \in \Psi_{+}$. The next statement is the relevant part of Theorem 2.2 of [16].

Proposition 3.1. Let $S_{0}$ be a positive $C_{0}$-semigroup on $\Psi$, with generator $\left(A_{0}, \mathcal{D}\left(A_{0}\right)\right)$. Let also $B: \mathcal{D}\left(A_{0}\right) \rightarrow \Psi$ be a positive linear operator such that

$$
\varphi\left(\left(A_{0}+B\right) \psi\right) \leq 0, \quad \psi \in \mathcal{D}\left(A_{0}\right) \cap \Psi_{+} .
$$

Then for all $r \in(0,1)$, the operator $\left(A_{0}+r B, \mathcal{D}\left(A_{0}\right)\right)$ is the generator of a substochastic semigroup on $\Psi$.

Assume that there exists a subspace $\widetilde{\Psi} \subset \Psi$ such that: (a) there exists a norm $\|\cdot\|_{\widetilde{\Psi}}$ and a positive linear functional $\tilde{\varphi}$ on $\widetilde{\Psi}$ such that $\|\psi\|_{\widetilde{\Psi}}=\tilde{\varphi}(\psi)$ for $\psi \in \widetilde{\Psi}_{+}:=\widetilde{\Psi} \cap \Psi_{+} ;(\mathrm{b})\left(\widetilde{\Psi},\|\cdot\|_{\widetilde{\Psi}}\right)$ is a Banach space; (c) the embedding $\widetilde{\Psi} \hookrightarrow \Psi$ is continuous. Let $S_{0}$ be as in Proposition 3.1. Assume that each $S_{0}(t), t>0$, leaves $\widetilde{\Psi}$ invariant and the restrictions $\widetilde{S}_{0}(t)$ of $S_{0}(t)$ to $\widetilde{\Psi}$ constitute a $C_{0}$-semigroup $\widetilde{S}_{0}$ on $\widetilde{\Psi}$, generated by the restriction of $A_{0}$ to

$$
\mathcal{D}\left(\widetilde{A}_{0}\right)=\left\{\psi \in \mathcal{D}\left(A_{0}\right) \cap \widetilde{\Psi}: A_{0} \psi \in \widetilde{\Psi}\right\} .
$$

For $B$ as in Proposition 3.1, assume additionally that $B: \mathcal{D}\left(\widetilde{A}_{0}\right) \rightarrow \widetilde{\Psi}$. The next statement is the relevant part of Theorem 2.7 of [16].

Proposition 3.2. Let the assumptions above including (3.1) be satisfied. Additionally, assume that $-A_{0}$ is positive and there exists positive $c$ and $\varepsilon$ such that the following holds

$$
\tilde{\varphi}\left(\left(A_{0}+B\right) \psi\right) \leq c\|\psi\|_{\widetilde{\Psi}}-\varepsilon\left\|A_{0} \psi\right\|_{\Psi}, \quad \psi \in \mathcal{D}\left(\widetilde{A}_{0}\right) \cap \Psi_{+} .
$$

Then the closure of $\left(A_{0}+B, \mathcal{D}\left(A_{0}\right)\right)$ in $\Psi$ is the generator of a substochastic semigroup $S$ in $\Psi$ that leaves $\widetilde{\Psi}$ invariant. If the equality in (3.1) holds, then $S$ is stochastic. 
3.2. The proof. Claim (a)

Set $\Psi=\mathcal{M}, \widetilde{\Psi}=\mathcal{M}^{(n)}$, see (2.17), and

$$
\widetilde{\varphi}(\mu)=\int_{\Gamma_{0}}|\gamma|^{n} \mu(d \gamma)
$$

Note that $\varphi(\mu)=\mu\left(\Gamma_{0}\right)$, cf. (2.13). Next, set

$$
\left(A_{0} \mu\right)(d \gamma)=-\Xi\left(\mathbb{R}^{d}, \gamma\right) \mu(d \gamma), \quad \mathcal{D}\left(A_{0}\right)=\mathcal{D}\left(L^{\mu}\right)
$$

where the latter set is defined in (2.14). The operator $\left(A_{0}, \mathcal{D}\left(A_{0}\right)\right)$ generates the semigroup $S_{0}$ on $\mathcal{M}$ defined by

$$
\left(S_{0}(t) \mu(d \gamma)=\exp \left(-t \Xi\left(\mathbb{R}^{d}, \gamma\right)\right) \mu(d \gamma),\right.
$$

which is clearly substochastic. Then the operator, cf. (2.8),

$$
(B \mu)(d \gamma)=\int_{\Gamma_{0}} \Xi(d \gamma, \eta) \mu(d \eta)
$$

is positive and the following holds, cf. (2.13) and (3.5),

$$
\|B \mu\|=\left\|A_{0} \mu\right\|=\int_{\Gamma_{0}} \Xi\left(\mathbb{R}^{d}, \gamma\right) \mu_{+}(d \gamma)+\int_{\Gamma_{0}} \Xi\left(\mathbb{R}^{d}, \gamma\right) \mu_{-}(d \gamma),
$$

that is, $B: \mathcal{D}\left(A_{0}\right) \rightarrow \mathcal{M}$. Moreover, for positive $\mu \in \mathcal{D}\left(A_{0}\right)$, we have

$$
\varphi\left(\left(A_{0}+B\right) \mu\right)=-\int_{\Gamma_{0}} \Xi\left(\mathbb{R}^{d}, \gamma\right) \mu(d \gamma)+\int_{\Gamma_{0}} \Xi\left(\mathbb{R}^{d}, \gamma\right) \mu(d \gamma)=0
$$

The set defined in (3.2) consists of those $\mu \in \mathcal{M}^{(n)}$ for which

$$
\int_{\Gamma_{0}}|\gamma|^{n} \Xi\left(\mathbb{R}^{d}, \gamma\right) \mu_{ \pm}(d \gamma)<\infty
$$

Then $B$ defined in (3.6) maps this set into $\mathcal{M}^{(n)}$. Hence, to use Proposition 3.2 it is left to check the validity of the corresponding version of (3.3), the left-hand side of which can be written in the form, cf. (3.4) and (1.2),

$$
\begin{aligned}
\tilde{\varphi}\left(\left(A_{0}+B\right) \mu\right) & =\int_{\Gamma_{0}} F(\gamma)\left(L^{\mu} \mu\right)(d \gamma)=\left\langle\left\langle F L^{\mu} \mu\right\rangle\right\rangle \\
& =\langle\langle L F \mu\rangle\rangle=\int_{\Gamma_{0}}(L F)(\gamma) \mu(d \gamma),
\end{aligned}
$$

where $F(\gamma)=|\gamma|^{n}$ and $L$ is given in (1.5). Thus, (3.3) now takes the form

$$
\int_{\Gamma_{0}}\left((L F)(\gamma)-c F(\gamma)+\varepsilon \Xi\left(\mathbb{R}^{d}, \gamma\right)\right) \mu(d \gamma) \leq 0
$$


which has to hold for all positive $\mu$ with the property (3.7), including $\mu=\delta_{\eta}$ for each $\eta \in \Gamma_{0}$. This amounts to the following

$$
\begin{aligned}
& -\sum_{x \in \gamma}\left[m+E^{-}(x, \gamma \backslash x)\right](F(\gamma)-F(\gamma \backslash x)-\varepsilon) \\
& \quad+\int_{\mathbb{R}^{d}} E^{+}(y, \gamma)(F(\gamma \cup y)-F(\gamma)+\varepsilon) d y \leq c F(\gamma),
\end{aligned}
$$

which has to hold for some positive $c$ and $\varepsilon$ and all $\gamma \in \Gamma_{0}$. For $F(\gamma)=|\gamma|^{n}$ and $\varepsilon=1$, we have that $F(\gamma)-F(\gamma \backslash x)-\varepsilon \geq 0$ for all $\gamma \neq \emptyset$. Then (3.8) is satisfied if the following holds, cf. (2.10),

$$
\left\langle a^{+}\right\rangle|\gamma|\left[(|\gamma|+1)^{n}-|\gamma|^{n}+1\right] \leq c|\gamma|^{n},
$$

which is the case for $c=\left\langle a^{+}\right\rangle 2^{n+1}$. Thus, by Proposition 3.2 the closure of $\left(A_{0}+B, \mathcal{D}\left(A_{0}\right)\right)$ as given in (3.5), (3.6), and (2.14) generates a stochastic semigroup $S$ on $\mathcal{M}$, which leaves invariant $\mathcal{M}^{(n)}$. Then the solution in question is obtained as $\mu_{t}=S(t) \mu_{0}$.

Claim (b)

In this case, we set $\widetilde{\Psi}=\mathcal{M}^{\beta}$ and find $\beta>0$ for which (3.8) holds with $F(\gamma)=\exp (\beta|\gamma|)$. For $\left\langle a^{+}\right\rangle=0$, we fix arbitrary $\beta>0$ and then take $\varepsilon \leq e^{\beta}-1$. In this case, (3.8) holds for each $c>0$ and $\gamma \in \Gamma_{0}$. For $\left\langle a^{+}\right\rangle>0$, (3.8) holds, with each $c>0$, for positive $\beta$ and $\varepsilon$ that satisfy

$$
\left\langle a^{+}\right\rangle e^{2 \beta}-\left(\left\langle a^{+}\right\rangle+m\right) e^{\beta}+m=: P\left(e^{\beta}\right) \leq-\varepsilon\left(\left\langle a^{+}\right\rangle+m\right) .
$$

The polynomial $P$ has two roots 1 and $m /\left\langle a^{+}\right\rangle$. Thus, for each fixed $\beta$ such that the following holds $1<e^{\beta}<m /\left\langle a^{+}\right\rangle$, one finds $\varepsilon$ for which also (3.9) holds. This completes the proof in this case.

Claim (c)

Fix $\beta^{*}>0$ such that $\mu_{0} \in \mathcal{M}_{+, 1}^{\beta^{*}}$. By claim (a) we already know that $\mu_{t}=S(t) \mu_{0}$ is the unique global solution of (2.8) in $\mathcal{M}$. For a given $\beta_{*} \in$ $\left(0, \beta^{*}\right)$, let us show that (2.8) has also a classical solution in $\mathcal{M}^{\beta_{*}}$ on the time interval $\left(0, T_{*}\right)$. Since $\mathcal{M}^{\beta_{*}} \subset \mathcal{M}$, this solution would coincide with $S(t) \mu_{0}$ and hence be in $\mathcal{M}_{+, 1}^{\beta_{*}}$.

Thus, we consider (2.8) in $\mathcal{M}^{\beta_{*}}$ with $\mu_{0} \in \mathcal{M}_{+, 1}^{\beta^{*}}$. We study this problem by means of the scale $\left\{\mathcal{M}^{\beta}: \beta \in\left[\beta_{*}, \beta^{*}\right]\right\}$ of Banach spaces. Note that the embedding $\mathcal{M}^{\beta} \hookrightarrow \mathcal{M}^{\beta^{\prime}}, \beta>\beta^{\prime}$, is dense and continuous. For such $\beta$, we define $\mathcal{M}^{\beta}$ by

$$
\varphi_{\beta}(\mu)=\int_{\Gamma_{0}} \exp (\beta|\gamma|) \mu(d \gamma)
$$

Let also $A_{0}$ be defined on $\mathcal{M}^{\beta}$ by (3.5) with domain

$$
\mathcal{D}_{\beta}\left(A_{0}\right)=\left\{\mu \in \mathcal{M}^{\beta}: \Xi\left(\mathbb{R}^{d}, \cdot\right) \mu \in \mathcal{M}^{\beta}\right\} .
$$


We split the remaining part of $L^{\mu}$ in (2.8) into two terms, $B$ and $C$, defined on $\mathcal{M}^{\beta}$ as follows

$$
(B \mu)(A)=\int_{\Gamma_{0}}\left(\sum_{x \in \gamma}\left[m+E^{-}(x, \gamma \backslash x)\right] \mathbb{I}_{A}(\gamma \backslash x)\right) \mu(d \gamma),
$$

and

$$
(C \mu)(A)=\int_{\Gamma_{0}}\left(\int_{\mathbb{R}^{d}} E^{+}(y, \gamma) \mathbb{I}_{A}(\gamma \cup y) d y\right) \mu(d \gamma) \quad A \in \mathcal{B}\left(\Gamma_{0}\right) .
$$

Let us show that $B: \mathcal{D}_{\beta}\left(A_{0}\right) \rightarrow \mathcal{M}^{\beta}$. To this end we take $\mu \in \mathcal{D}_{\beta}\left(A_{0}\right) \cap \mathcal{M}_{+}$ and calculate, see (3.12), (2.11), and (2.12),

$$
\begin{aligned}
\|B \mu\|_{\mathcal{M}^{\beta}} & =\varphi_{\beta}(B \mu) \\
& =\int_{\Gamma_{0}} \exp (\beta|\eta|) \int_{\Gamma_{0}}\left(\sum_{x \in \gamma}\left[m+E^{-}(x, \gamma \backslash x)\right] \delta_{\eta \backslash x}(d \eta)\right) \mu(d \gamma) \\
& =e^{-\beta} \int_{\Gamma_{0}} \exp (\beta|\gamma|)\left(m|\gamma|+E^{-}(\gamma)\right) \mu(d \gamma) \\
& \leq e^{-\beta} \int_{\Gamma_{0}} \exp (\beta|\gamma|) \Xi\left(\mathbb{R}^{d}, \gamma\right) \mu(d \gamma) .
\end{aligned}
$$

The latter estimate and (3.5) and (3.10) yield

$$
\varphi_{\beta}\left(\left(A_{0}+r B\right) \mu\right) \leq 0, \quad \mu \in \mathcal{D}_{\beta}\left(A_{0}\right) \cap \mathcal{M}_{+},
$$

holding for some $r \in(0,1)$. Hence, by Proposition $3.1\left(\left(A_{0}+B\right), \mathcal{D}_{\beta}\left(A_{0}\right)\right)$ is the generator of a substochastic semigroup $S_{\beta}$ on $\mathcal{M}^{\beta}$. Now for $\beta^{\prime} \in\left(\beta_{*}, \beta\right.$, let us show that $C$ as given in (3.13) acts as a bounded linear operator $C: \mathcal{M}^{\beta} \rightarrow \mathcal{M}^{\beta^{\prime}}$. To this end, as in (3.14) we take $\mu \in \mathcal{D}_{\beta}\left(A_{0}\right) \cap \mathcal{M}_{+}$and calculate, see (2.11),

$$
\begin{aligned}
\|C \mu\|_{\mathcal{M}^{\beta^{\prime}}} & =\varphi_{\beta^{\prime}}(C \mu) \\
& =\int_{\Gamma_{0}} \exp \left(\beta^{\prime}|\eta|\right) \int_{\Gamma_{0}}\left(\int_{\mathbb{R}^{d}} E^{+}(y, \gamma) \delta_{\gamma \cup y}(d \eta) d y\right) \mu(d \gamma) \\
& =e^{\beta^{\prime}}\left\langle a^{+}\right\rangle \int_{\Gamma_{0}} \exp \left(\beta^{\prime}|\gamma|\right)|\gamma| \mu(d \gamma) \\
& \leq \frac{e^{\beta^{\prime}}\left\langle a^{+}\right\rangle}{e\left(\beta-\beta^{\prime}\right)} \int_{\Gamma_{0}} \exp (\beta|\gamma|) \mu(d \gamma)=\frac{e^{\beta}\left\langle a^{+}\right\rangle}{e\left(\beta-\beta^{\prime}\right)}\|\mu\|_{\mathcal{M}^{\beta}} .
\end{aligned}
$$

That is, the operator norm $\|C\|_{\beta, \beta^{\prime}}$ of $C: \mathcal{M}^{\beta} \rightarrow \mathcal{M}^{\beta^{\prime}}$ satisfies

$$
\|C\|_{\beta, \beta^{\prime}} \leq \frac{e^{\beta}\left\langle a^{+}\right\rangle}{e\left(\beta-\beta^{\prime}\right)} .
$$


In obtaining the last line in (3.15), we have used the following obvious inequality

$$
\exp \left[-\left(\beta-\beta^{\prime}\right) N\right] N \leq \frac{1}{e\left(\beta-\beta^{\prime}\right)}, \quad N \in \mathbb{N}
$$

For fixed $n \in \mathbb{N}$ and $\beta \in\left(\beta_{*}, \beta^{*}\right)$, set

$$
\begin{aligned}
& \mu_{t}^{(n)}=S_{\beta}(t) \mu_{0}+\sum_{l=1}^{n} \int_{0}^{t} \int_{0}^{t_{1}} \cdots \int_{0}^{t_{l-1}} T_{l}\left(t, t_{1}, \ldots, t_{l}\right) \mu_{0} d t_{l} \cdots d t_{1} \\
& T_{l}\left(t, t_{1}, \ldots, t_{l}\right)=S_{\beta_{l}}\left(t-t_{1}\right) C_{\beta_{l}} S_{\beta_{l-1}}\left(t_{1}-t_{2}\right) \cdots S_{\beta_{l}}\left(t_{l-1}-t_{l}\right) C_{\beta_{1}} S_{\beta_{0}}\left(t_{l}\right),
\end{aligned}
$$

where, for a fixed $l \geq 1$,

$$
\beta_{s}=\beta^{*}-s \epsilon, \quad \epsilon=\left(\beta^{*}-\beta\right) / l, \quad s=0, \ldots, l,
$$

and hence $\beta_{0}=\beta^{*}$ and $\beta_{l}=\beta$. For $s=0, \ldots, l$, the operator $C_{\beta_{s}}$ acts from $\mathcal{M}^{\beta_{s-1}}$ to $\mathcal{M}^{\beta_{s}}$ as a bounded operator, see (3.15) and (3.16). Note that $\mu_{t}^{(n)} \in \mathcal{M}^{\beta}$ for each $t>0$ and $n \in \mathbb{N}$. Note also that each $T_{l}$ is continuous in $t$ and each of $t_{s}, s=1, \ldots, l$, which follows from the strong continuity of each $S_{\beta_{s}}$. This yields that $\mu_{t}^{(n)}$ is continuously differentiable in $\mathcal{M}^{\beta}$. Furthermore, for each $\beta^{\prime} \in\left[\beta_{*}, \beta\right)$, we have that the derivative as an element of $\mathcal{M}^{\beta^{\prime}}$ satisfies

$$
\frac{d}{d t} \mu_{t}^{(n)}=\left(A_{0}+B\right) \mu_{t}^{(n)}+C \mu_{t}^{(n-1)}
$$

where all these operators act in $\mathcal{M}^{\beta^{\prime}}$ with domains containing $\mathcal{M}^{\beta}$, see (3.11), (3.15), and (3.16).

By (3.17) and (3.18) we have

$$
\begin{aligned}
\left\|\mu_{t}^{(n)}-\mu_{t}^{(n-1)}\right\|_{\mathcal{M}^{\beta}} \leq & \int_{0}^{t} \int_{0}^{t_{1}} \cdots \int_{0}^{t_{l-1}}\left\|T_{l}\left(t, t_{1}, \ldots, t_{l}\right) \mu_{0}\right\|_{\mathcal{M}^{\beta}} d t_{l} \cdots d t_{1} \\
& \leq \frac{t^{n}}{n !}\left\|C_{\beta_{n}} C_{\beta_{n-1}} \cdots C_{\beta_{1}} \mu_{0}\right\|_{\beta} \\
\leq & \frac{t^{n}}{n !}\left(\prod_{s=1}^{n}\left\|C_{\beta_{s}}\right\|_{\beta_{s-1}, \beta_{s}}\right)\left\|\mu_{0}\right\|_{\beta^{*}} \\
& \leq \frac{1}{n !}\left(\frac{n}{e}\right)^{n}\left(\frac{t e^{\beta^{*}}\left\langle a^{+}\right\rangle}{\beta^{*}-\beta}\right)^{n}
\end{aligned}
$$


By (3.2) the sequence $\left\{\mu_{t}^{(n)}\right\}_{n \in \mathbb{N}}$ converges in $\mathcal{M}^{\beta}$ to a certain $\mu_{t}$, uniformly on each $[0, \theta], \theta<T\left(\beta^{*}, \beta\right)$. On the other hand,

$$
\begin{aligned}
& \sup _{t \in[0, \theta]}\left\|\left(A_{0}+B\right)\left[\mu_{t}^{(n)}-\mu_{t}^{(n-1)}\right]\right\|_{\mathcal{M}^{\beta^{\prime}}} \\
& \leq\left(\left\|A_{0}\right\|_{\beta, \beta^{\prime}}+\|B\|_{\beta, \beta^{\prime}}\right) \sup _{t \in[0, \theta]}\left\|\mu_{t}^{(n)}-\mu_{t}^{(n-1)}\right\|_{\mathcal{M}^{\beta}}, \\
& \sup _{t \in[0, \theta]}\left\|C\left[\mu_{t}^{(n)}-\mu_{t}^{(n-1)}\right]\right\|_{\mathcal{M}^{\beta^{\prime}}} \leq\|C\|_{\beta, \beta^{\prime}} \sup _{t \in[0, \theta]}\left\|\mu_{t}^{(n)}-\mu_{t}^{(n-1)}\right\|_{\mathcal{M}^{\beta}},
\end{aligned}
$$

where the operator norms can be estimated as in (3.16). Hence, by (3.2) $\left\{d \mu_{t}^{(n)} / d t\right\}_{n \in \mathbb{N}}$ converges in $\mathcal{M}^{\beta^{\prime}}$, uniformly on $[0, \theta]$. Therefore, the limiting $\mu_{t} \in \mathcal{M}^{\beta^{\prime}}$ is continuously differentiable on each $[0, \theta] \subset\left[0, T\left(\beta^{*}, \beta^{\prime}\right)\right)$, and

$$
\mu_{t}^{(n)} \rightarrow \mu_{t} \in \mathcal{M}^{\beta^{\prime}}, \quad n \rightarrow+\infty .
$$

On the other hand, the right-hand side of (3.19) converges in $\mathcal{M}^{\beta^{\prime}}$ to $L^{\mu} \mu_{t}$. Hence, the limiting $\mu_{t}$ is the classical solution of (2.8) in $\mathcal{M}^{\beta^{\prime}}$ on the time interval $\left[0, T\left(\beta^{*}, \beta^{\prime}\right)\right)$. Now we set $\beta^{\prime}=\beta_{*}$ and obtain the proof for this case.

Claim (d)

We consider the problem in (2.16) and repeat the arguments used in the proof above. By the uniqueness of the solution of (2.8) we obtain that $\mu_{t}$ in Claims (a) - (c) and $\mu_{t}:=R_{t} \lambda$ coincide, which yields the proof.

Acknowledgement: The author thanks Yuri Kondratiev for fruitful discussions on the subject of this work.

\section{REFERENCES}

[1] Albeverio, S., Kondratiev, Yu. G., and Röckner, M.: Analysis and geometry on configuration spaces. J. Func. Anal. 154, 444-500 (1998)

[2] Dobrushin, R. L., Sinai, Y. G., and Sukhov, Y. M.: Dynamical systems of statistical mechanics. In: Itogi Nauki, pp. 235-284. VINITI (1985); eng. transl. in: Sinai Ya. G. (ed.) Ergodic Theory with Applications to Dynamical Systems and Statistical Mechanics, II, Encyclopaedia Math. Sci., Springer, Berlin Heidelberg 1989

[3] Finkelshtein, D., Kondratiev, Y., Kozitsky, Y., and Kutoviy, O.: Stochastic evolution of a continuum particle system with dispersal and competition. Eur. Phys. J. Special Topics 216, 107-116 (2103)

[4] Finkelshtein, D., Kondratiev, Y., Kozitsky, Y., and Kutoviy, O.: The statistical dynamics of a spatial logistic model and the related kinetic equation. arXiv:1401.0557v1, 2014 (to appear in Math. Models Methods Appl. Sci.)

[5] Finkelshtein, D., Kondratiev, Y., and Kutoviy, O.: An operator approach to Vlasov scaling for some models of spatial ecology. Methods Funct. Anal. Topology 19108 126 (2013)

[6] Finkelshtein, D., Kondratiev, Y., and Kutoviy, O.: Semigroup approach to birthand-death stochastic dynamics in continuum. J. Funct. Anal. 262, 1274-1308 (2012)

[7] Finkelshtein, D. L., Kondratiev, Yu. G., and Kutoviy, O.: Individual based model with competition in spatial ecology. SIAM J. Math. Anal. 41, 297-317 (2009) 
[8] Finkelshtein, D. L., Kondratiev, Yu. G., and Oliveira, M. J.: Markov evolution and hierarchical equations in the continuum. I: One-component systems. J. Evol. Equ. 9 197-233 (2009)

[9] Fournier, N. and Méléard, S.: A microscopic probabilistic description of a locally regulated population and macroscopic approximations. Ann. Appl. Probab. 14 18801919 (2004)

[10] Kondratiev, Yu. and Kuna, T.: Harmonic analysis on configuration space. I. General theory. Infin. Dimens. Anal. Quantum Probab. Relat. Top. 5, 201-233 (2002)

[11] Kondratiev, Yu, Kutoviy, O, and Pirogov, S.: Correlation functions and invariant measures in continuous contact model. Infin. Dimens. Anal. Quantum Probab. Relat. Top. 11, 231-258 (2008)

[12] Kondratiev, Yu. G. and Skorokhod, A. V. On contact models in continuum. Infin. Dimens. Anal. Quantum Probab. Relat. Top. 9, 187-198 (2006)

[13] Neuhauser, C.: Mathematical challenges in spatial ecology. Notices of AMS. 48 (11), 1304-1314 (2001)

[14] Ovaskainen, O., Finkelshtein, D., Kutoviy, O, Cornell, S, Bolker, B, and Kondratiev, Yu.: A general mathematical framework for the analysis of spatiotemporal point processes. Theor. Ecol. 7, 101-113 (2014)

[15] Parthasarathy, K.P. (1967) Probability Measures on Metric Spaces. New York - London: Probability and Mathematical Statistics 3, Academic Press, Inc.

[16] Thieme, H.R. and Voigt, J.: Stochastic semigroups: their construction by perturbation and approximation. In Positivity IVtheory and applications, 135-146, Tech. Univ. Dresden, Dresden, 2006

Institute of Mathematics, Maria Curie-Skeodowska University, Lublin 20031 POLAND

E-mail address: jkozi@hektor.umcs.lublin.pl 